\title{
MÉTODOS DE ELIMINAÇÃO DE SOLUÇÕES ESPÚRIAS DE GUIAS DIELÉTRICOS POR ELEMENTOS FINITOS : COMPARAÇÃO ENTRE DESEMPENHOS COMPUTACIONAIS
}

\author{
C. G. Migliora \\ CETUC - Centro de Estudos em Telecomunicações - PUC-Rio \\ M. B. F. Chaves e H. J. C. Barbosa \\ LNCC - Laboratório Nacional de Computação Científica - CNPq
}

Resumo: Três métodos de eliminação de modos espúrios em soluções de guias de ondas dielétricos, inomogêneos e/ou anisotrópicos, usando formulaçōes variacionais e o Métodos de Elementos Finitos são analisados neste trabalho. O método da penalidade com integração reduzida, recentemente aplicado a problemas de Eletromagnetismo, tem o seu desempenho computacional comparado com $o$ do método das componentes transversais de Hayata e Koshiba e com o de uma abordagem que emprega elementos de aresta. A qualidade das aproximações e as características computacionais comprovam a eficiência da integração reduzida, suprimindo os principais inconvenientes do método da penalidade e mantendo os seus atrativos e a simplicidade da implementação.

\begin{abstract}
Three methods for elimination of spurious modes from variationally formulated Finite Element $\therefore$ itions of inhomogeneous and/or anisotropic dielectric waveguides are considered. The reduced integration penalty method, recently applied to EM wave problems, is compared, in terms of computational performance, to the method of transversal components by Hayata and Koshiba and to an edge element based approach. The quality of the spproximations obtained and its computational characteristics testify the efficiency of the reduced integration in suppressing the main drawbacks of the penalty method, while preserving its advantages and simple implementation.
\end{abstract}

Palavras Chave: Guias Dielétricos, Modos Espúrios, Elementos Finitos, Penalidade com Integração Reduzida.

\section{INTRODUÇÃO}

As primeiras aplicações do Método de Elementos Finitos (MEF) em problemas de Eletromagnetismo (EM) surgiram na literatura no final da década de $60 \mathrm{e}$, desde

Este trabalho foi parcialmente financiado pela TELEBRÁS através do contrato PUC/TELEBRÁS JDPqD-513 / 93.

Publicação recomendada pelo Editor de Área de Microondas e Optoeletrônica. então, o número de publicações no assunto vem crescendo exponencialmente: do primeiro, em 1968, a quase $700 \mathrm{em}$ 1990, são mais de 5000 trabalhos que comprovam o alcance do método [1]. No estudo de guias óticos e de microondas, o MEF tem aparecido com muita ênfase, principalmente a partir da década de 80 , quando se estabeleceu como "provavelmente, a mais poderosa e eficiente solução numérica do problema mais geral de guias de ondas óticos" [2].

No caso de guias de ondas homogêneos e isotrópicos, onde a propagação pode ser descrita em termos de modos TE e TM, a formulação variacional que serve de base para a aplicação do MEF é escalar e se expressa, geralmente, em termos das componentes longitudinais desses modos. Quando o guia apresenta anisotropia e/ou inomogeneidade transversal, os modos híbridos de propagação tornam necessária a utilização de uma forma variacional vetorial associada ao método. Neste caso, uma das maiores dificuldades que surgem no cálculo numérico das características de propagação é o aparecimento de soluções não-físicas, os chamados modos espúrios, relatado desde as primeiras tentativas de resolver modelos vetoriais $[3,4]$.

Nas aplicaçōes do MEF a guias de ondas dielétricos em que a anisotropia e/ou a inomogeneidade se devem à permissividade elétrica, a formulação variacional mais apropriada é expressa em termos do vetor campo magnético, e os modos espúrios são as soluções do problema discretizado que violam a condição de solenoidalidade de $\mathbf{H}$. O espaço das aproximações gerado pela forma variacional discretizada não corresponde ao espaço das soluções da equação diferencial original e obtem-se, assim, pares de autovalores e autovetores nãofísicos.

Ao longo de mais de duas décadas, vários autores têm tentado explicar as origens desse problema e buscado formas de garantir a confiabilidade das soluções obtidas. Inicialmente, o procedimento consistia na identificação $a$ posteriori das soluções espúrias e sua consequente remoção. As dificuldades de automatização destes processos, além de seu elevado custo computacional, levaram à investigação de métodos para eliminar a priori os autopares espúrios. 
Neste trabalho, os diferentes procedimentos propostos visando a eliminação a priori de modos espúrios em problemas de ondas guiadas, foram classificados em 3 categorias distintas, a saber: modificação do funcional básico, uso de outras formulações variacionais e uso de novos elementos. De cada uma dessas categorias, foi escolhido um método para ser implementado, com o objetivo de se efetuar uma comparação entre seus desempenhos computacionais.

Da primeira categoria, foi implementado o método da penalidade [5,6], com uma modificação recentemente introduzida $[7,8]$ que permite pré-fixar o valor do parâmetro de penalidade, garantindo a eliminação dos modos espúrios do intervalo espectral de interesse sem afetar significativamente a precisão dos modos físicos. Este método, chamado de penalidade com integração reduzida, tem implementação simples e mantém a esparsidade das matrizes, uma característica computacional importante do método da penalidade. Dentro da segunda categoria, foi escolhido o método das componentes transversais de Hayata e Koshiba [9], devido à simplicidade de sua formulação teórica e por eliminar os modos espúrios de todo o espectro.

Na terceira categoria, a denominação genérica "elementos vetoriais tangenciais" caracteriza a classe cujas funções de base têm os graus de liberdade associados às arestas e não aos vértices dos elementos, como nos tradicionais elementos Lagrangeanos. Nesta classe, cuja propriedade mais importante é a continuidade imposta apenas à componente tangencial do campo, o conjunto mais simples é composto pelos elementos de aresta (edge elements). $\mathrm{Na}$ formulação aqui implementada, a componente axial do campo - que deve ser sempre contínua nas interfaces entre materiais diferentes - foi aproximada por elementos nodais, mas para a componente transversal - que pode apresentar descontinuidade normal nessas interfaces - foram usados elementos de aresta. Com isso, é possível impor aos campos apenas a continuidade fisicamente requerida, por meio de uma formulação simples e eficiente. Através dos resultados obtidos em diversos experimentos numéricos, busca-se identificar as vantagens e as desvantagens dessas três abordagens, no sentido de garantir a confiabilidade das soluções. Em particular, comprova-se a eficiência e a simplicidade do método da penalidade com integração reduzida, analisando-se seu desempenho computacional em comparação com outros já estabelecidos.

Na seção 2, a formulação em termos do campo vetorial magnético para o problema de autovalores de guias de ondas longitudinalmente uniformes é apresentada e, em seguida, desenvolve-se a forma variacional equivalente e a sua discretização pelo MEF. Na seção 3, após uma discussão geral sobre a eliminação de modos espúrios em guias de ondas, são descritos os métodos escolhidos para ser implementados, obtendo-se, para cada um deles, a equação matricial global do MEF. A seção 4 descreve brevemente as implementações computacionais e sua validação, apresentando, em seguida, os resultados obtidos com os métodos de eliminação de espúrios para alguns tipos de guias de ondas dielétricos. Compara-se, então, o desempenho destes métodos em termos de precisão, tempo de computação e quantidade de memória requerida, concluindo-se as vantagens relativas de cada um.

\section{FORMULAÇÃO DO PROBLEMA}

\subsection{O Problema de Autovalores}

Seja um guia de ondas sem fontes, uniforme na direção z e com seção reta arbitrária $\Omega$ no plano xy, como mostra a ura 1 , onde $\varepsilon$ e $\mu$ são, respectivamente, a permissividade elétrica e a permeabilidade magnética relativas do meio interno ao guia, e uma dependência temporal harmônica da forma $\exp (j w t)$ è assumida, sendo $\omega$ a freqüência angular. A fronteira do guia se particiona, no caso mais geral, em um condutor elétrico perfeito $\left(\delta \Omega_{E}\right)$ e um condutor magnético perfeito $\left(\delta \Omega_{\mathrm{M}}\right)$. Devido ao interesse em aplicações a guias inomogêneos e anisotrópicos, assumindo $\mu=1$ e $\varepsilon=\varepsilon(\mathrm{x}, \mathrm{y})$ como $\mathrm{o}$ tensor permissividade relativa, obtem-se das equações de Maxwell a equação vetorial de Helmholtz para o campo magnético:

$$
\operatorname{rot}\left[\varepsilon^{-1} \operatorname{rot} \mathbf{H}\right]-\mathrm{k}_{0}^{2} \mathbf{H}=0
$$

sendo $k_{0}=\omega\left(\mu_{0} \varepsilon_{0}\right)^{1 / 2}$ o número de onda no espaço livre e tendo como condições de contorno:

$$
\begin{gathered}
\mathbf{n} \times \mathbf{H}=0 \quad \text { em } \\
\mathbf{n} \times\left(\varepsilon^{-1} \text { rot } \mathbf{H}\right)=0 \quad \text { em } \delta \Omega_{\mathrm{E}}
\end{gathered}
$$

onde $\mathbf{n}$ denota o vetor unitário normal à fronteira. $\mathrm{Na}$ interface entre dois materiais distintos, identificados pelos índices $\mathrm{i}$ e j, o campo magnético deve satisfazer ainda às condiçōes de continuidade:

$$
\begin{gathered}
\mathbf{n} \times\left(\mathbf{H}_{\mathbf{i}}-\mathbf{H}_{\mathbf{j}}\right)=0 \\
\mathbf{n} \times\left(\varepsilon_{\mathbf{i}}^{-1} \operatorname{rot} \mathbf{H}_{\mathbf{i}}-\varepsilon_{\mathrm{j}}^{-1} \operatorname{rot} \mathbf{H}_{\mathbf{j}}\right)=0
\end{gathered}
$$

sendo $\mathbf{n}$, aqui, o vetor unitário normal à interface entre os meios. A condição de solenoidalidade do campo magnético está implícita em (1) quando $\mathrm{k}_{0}{ }^{2} \quad 0$, o que

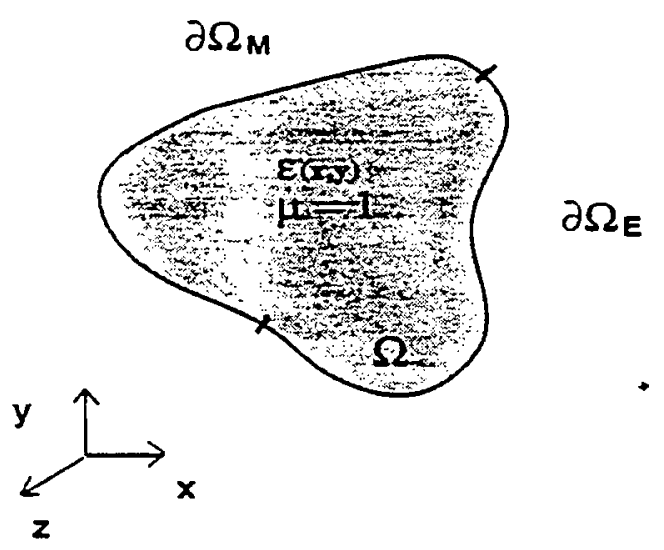

Figura 1 - Geometria da Seção reta (arbitrária) do guia de ondas. 
pode ser facilmente comprovado tomando-se o divergente daquela equação, ou seja, as soluçōes de (1) com $k_{0}^{2} 0$, também satisfazem a div $\mathbf{H}=0$.

A equação (1) tem soluções não-triviais apenas para uma coleção enumerável de autovalores $\left\{\lambda_{j}=k_{0 j}{ }^{2}\right\}$,e a coleção $\left\{\mathbf{v}_{\mathbf{j}}=\mathbf{H}_{\mathbf{j}}\right\}$ das autofunções normalizadas correspondentes forma um conjunto ortonormal completo em $\left\{L_{2}(\Omega)\right\}^{3}$, onde $L_{2}(\Omega)$ é o espaço de Hilbert de funções de quadrado integrável definidas em $\Omega$. A determinação dos autopares $\left(\lambda_{j}, \mathbf{v}_{j}\right)$ constitui o problema de autovalores (PAV) associado a (1), (2) e (3), definido de forma mais compacta como:

\section{(PAV)}

Achar $(\lambda, \mathbf{v})$ tal que $\mathbf{v} E S(\operatorname{rot}, \Omega), \mathbf{v} 0$ e $\operatorname{div} \mathbf{v}=0 \mathrm{em} \Omega$, $\mathrm{L} \mathbf{v}=\lambda \mathbf{v}$ em $\Omega, \mathbf{n} \times \mathbf{v}=0 \mathrm{em} \delta \Omega_{\mathrm{E}}$ e $\mathbf{n} \times\left(\varepsilon^{-1} \operatorname{rot} \mathbf{v}\right)=0$ em $\delta \Omega_{\mathrm{M}}$

onde

$$
\begin{aligned}
& \lambda=\mathrm{k}_{0}{ }^{2}, \mathrm{Lv}=\operatorname{rot}\left(\varepsilon^{-1} \operatorname{rot} \mathbf{v}\right) \mathrm{e} \\
& \mathrm{S}(\operatorname{rot}, \Omega)=\left\{\mathrm{v} E\left(\mathrm{~L}_{2}(\Omega)\right)^{3} / \mathrm{L} \vee \mathrm{E}\left(\mathrm{L}_{2}(\Omega)\right)^{3},\right. \\
& \mathbf{n} \times \mathbf{v}=0 \mathrm{em} \delta \Omega_{\mathrm{E}} \\
&\left.\mathbf{n} \times\left(\varepsilon^{-1} \operatorname{rot} \mathbf{v}\right)=0 \mathrm{em} \delta \Omega_{\mathrm{M}}\right\}
\end{aligned}
$$

Seria possível, em alguns casos, obter soluções aproximadas diretamente do PAV, como no Método de Diferenças Finitas, mas a abordagem utilizada aqui será a do $\mathrm{MEF}$, que se baseia na formulação variacional (ou fraca) descrita a seguir. Esta opção se define, basicamente, na escolha do esquema de computação que se deseje aplicar.

\subsection{Formulação Variacional}

Seja v uma solução forte (ou clássica) do PAV representado em (6). Então:

$$
(\mathbf{L} \mathbf{v}, \mathbf{w})=\lambda(\mathbf{v}, \mathbf{w}), \mathbf{w} \mathrm{E}(\operatorname{rot}, \Omega)
$$

onde (...) denota o produto interno em $L_{2}(\Omega)$ e w representa as chamadas funções teste admissíveis que, por pertencerem a $S$ ( rot, $\Omega$ ), satisfazem às mesmas condições de contorno da solução. Nas aplicações do MEF a problemas de Eletromagnetismo, o PAV a ser resolvido é, inicialmente, substituído por uma formulação fraca (ou variacional) equivalente para a qual se busca, então, uma solução aproximada. No caso do PAV formulado em (6), o uso do produto interno complexo em (7) e a aplicação de algumas identidades vetoriais leva à formulação fraca [7] :

Achar $\{\lambda, v\} E R \times V(\operatorname{rot}, \Omega)$ tal que $v 0$ e

$$
\left(\varepsilon^{-1} \operatorname{rot} \mathbf{v}, \operatorname{rot} \mathbf{w}\right)=\lambda(\mathbf{v}, \mathbf{w}), \quad w E V(\operatorname{rot}, \Omega)
$$

onde

$$
\begin{aligned}
\mathrm{V}(\operatorname{rot}, \Omega)= & \left\{\mathrm{vE}\left(\mathrm{L}_{2}(\Omega)\right)^{3} / \operatorname{rot} \mathbf{v E}\left(\mathrm{L}_{2}(\Omega)\right)^{3},\right. \\
& \left.\mathbf{n} \cdot \mathbf{v}=0 \text { em } \delta \Omega_{\mathrm{E}} \text { e } \mathbf{n x v}=0 \text { em } \delta \Omega_{\mathrm{M}}\right\}
\end{aligned}
$$

Esta formulação facilita a obtenção de soluçōes aproximadas por ser, na grande maioria dos problemas, mais simples de tratar numericamente do que a equação original . O procedimento geral consiste em escolher um subespaço de dimensão finita $V_{h}$

$\mathrm{V}$ e nele buscar as soluções de (8). O MEF, descrito a seguir, se encarrega de construir este subespaço.

\subsection{Discretização por Elementos Finitos}

A formulação fraca pode ser usada para obter soluçōes aproximadas para o PAV, uma vez escolhido o conjunto de funçōes teste conveniente. O método de Rayleigh-Ritz [10] consiste em escolher um número finito de funçōes teste $\mathbf{a}_{\mathbf{k}}, \mathrm{k}=1, \ldots, \mathrm{N}$ e, dentre todas as suas possíveis combinações lineares, identificar aquela que torna $O$ funcional estacionário. Na chamada aproximação de Ritz, os coeficientes desconhecidos são determinados por um sistema de equaçōes, e a escolha das funçōes teste irá definir a major ou menor complexidade na construção desse sistema, especialmente quando o problema envolve domínios irregulares e condições de contorno não-triviais.

A aproximaçāo de Galerkin é a mais usual dessas técnicas de discretização e se caracteriza pela escolha do mesmo espaço $V_{h}$ (de dimensão finita) para aproximar as soluções $\mathrm{e}$ as funçōes teste. O MEF fornece uma técnica conveniente para a geração sistemática das funções $\mathbf{a}_{\mathbf{k}}$ do espaço de aproximação. Consiste, inicialmente, em particionar a região estudada $\Omega$ num conjunto de $\mathrm{E}$ elementos finitos $\Omega_{\mathbf{e}}$

$$
\Omega=\cup_{1}^{E} \quad \Omega_{\mathrm{e}}, \quad \Omega_{\mathrm{i}} \cap \Omega_{\mathrm{j}} \neq \varnothing \quad \text { para } \mathrm{i} \neq \mathrm{j}
$$

$O$ conjunto finito de funções teste $a_{k}$ gera $V_{h}$ que, por sua vez, é subespaço de $\mathrm{V}$. $\mathrm{O}$ índice $\mathrm{h}$ está associado ao tamanho $\mathrm{e}$, consequentemente, ao número total $\mathrm{E}$ de elementos usados para discretizar $\Omega$. Uma vez escolhido o espaço de funções de interpolação $\mathbf{a}_{\mathbf{k}} E \mathrm{~V}_{\mathrm{h}}$, cada componente da solução fraca é aproximada pela combinação linear:

$$
\mathbf{v}_{\mathrm{h}}=\sum_{\mathrm{k}=1}^{\mathrm{N}} \mathrm{q}_{\mathrm{k}} \mathbf{a}_{\mathrm{k}}
$$

Usando o mesmo conjunto de funçōes para as funçōes teste w (Galerkin), é possível escrever a formulação (8) discretizada por elementos finitos:

Achar $\left\{\left(\mathrm{k}_{0}^{2}\right)_{\mathrm{h}}, \mathbf{v}_{\mathrm{h}}\right\} \mathrm{E} \times \mathrm{V}_{\mathrm{h}}$ tal que $\mathbf{v}_{\mathbf{h}} \neq 0 \mathrm{e}$ $\left(\varepsilon^{-1} \operatorname{rot} \mathbf{v}_{h}, \operatorname{rot} \mathbf{w}_{h}\right)=\left(k_{0}^{2}\right)_{h}\left(\mathbf{v}_{h}, \mathbf{w}_{h}\right), \quad \mathbf{w}_{h} E V_{h}$

No problema considerado, a onda EM propaga-se na direção $z$ com um campo magnético da forma:

$$
\mathbf{H}(\mathrm{x}, \mathrm{y}, \mathrm{z})=\mathbf{H}(\mathrm{x}, \mathrm{y}) \exp (-j \beta z
$$

e a solução $\mathbf{v}=\mathrm{HE} \mathrm{V}$ ( $\operatorname{rot}, \Omega)$ do PAV em (6) coincide com o ponto estacionário do funcional:

$$
\mathrm{F}(\mathrm{H})=\int_{\Omega}\left[\operatorname{rot} \mathbf{H}^{*} \cdot\left(\varepsilon^{-1} \operatorname{rot} \mathbf{H}\right)-\mathrm{k}_{0}{ }^{2} \mathbf{H}^{*} \cdot \mathbf{H}\right] d \Omega
$$


associado a (8), com $\mathbf{w}=\mathrm{H} E \mathrm{~V}(\operatorname{rot}, \Omega)$. Dividindo a seção reta $\Omega$ do guia em elementos com n nós, o campo magnético em cada elemento pode ser escrito em termos dos seus valores nodais:

onde

$$
\mathbf{H}_{\mathrm{e}}=[\mathrm{N}]^{\mathrm{T}} \mathbf{h}_{\mathrm{e}} \exp (-j \beta z
$$

$$
[N]=\left[\begin{array}{ccc}
\mathrm{Ne} & 0 & 0 \\
0 & \mathrm{Ne} & 0 \\
0 & 0 & \mathrm{jNe}
\end{array}\right]
$$

$\mathrm{e}$

$$
h_{e}=\left[\begin{array}{lll}
h_{x} & h_{y} & h_{z}
\end{array}\right]
$$

Aqui, $\mathbf{N}_{\mathrm{e}}$ representa o vetor das funções de base nodais, $\mathbf{h}_{\mathbf{x}}, \mathbf{h}_{\mathbf{y}}$ e $\mathbf{h}_{\mathbf{z}}$ são os vetores formados pelos $n$ valores nodais das componentes do campo vetorial magnético em cada elemento, 0 é o vetor nulo e $T$ denota transposição. Usando-se a expressão aproximada para $\mathrm{H}$ em (14) no funcional (13) e as técnicas usuais do MEF [11], obtém-se a equação matricial global:

$$
\begin{gathered}
{[\mathrm{S}] \mathbf{h}-\mathrm{k}_{0}^{2}[\mathrm{~T}] \mathbf{h}=0} \\
{[\mathrm{~S}]=\sum_{\mathbf{e}} \int_{\Omega e}[\mathrm{~B}]^{\star} \varepsilon^{-1}[\mathrm{~B}]^{\mathrm{T}} d \mathrm{x} d \mathrm{y}} \\
{[\mathrm{T}]=\sum_{\mathbf{e}} \int_{\Omega e}[N]^{\star}[N]^{\mathrm{T}} d \mathrm{x} d \mathrm{y}} \\
{[\mathrm{B}]=\left[\begin{array}{ccc}
0 & -\mathrm{j} \beta \mathrm{N}_{\mathrm{e}} & -d \mathrm{~N}_{e} / d \mathrm{y} \\
\mathrm{j} \beta \mathrm{N}_{\mathrm{e}} & 0 & d \mathrm{~N}_{\mathrm{e}} / d \mathrm{x} \\
d \mathrm{~N}_{\mathrm{e}} / d \mathrm{y} & -d \mathrm{~N}_{\mathrm{e}} / d \mathrm{x} & 0
\end{array}\right]}
\end{gathered}
$$

h é o vetor formado pelos valores nodais das componentes do campo vetorial magnético em $\Omega$ e $\sum_{e}$ simboliza o processo de soma das contribuições de cada elemento nas matrizes globais [S] e [T]. Vale ressaltar que desenvolvimento análogo pode ser feito para o campo elétrico, chegando-se ao mesmo tipo de sistema matricial. Infelizmente, esta formulação gera soluçōes espúrias, que violam a condição de solenoidalidade do campo $\mathbf{H}$, entremeadas às soluções corretas, correspondentes aos modos físicos do guia considerado. A seguir, serão analisadas técnicas para tratar esse problema.

\section{MÉTOdOS DE ELIMINAÇÃO DOS MODOS ESPÚRIOS}

Desde as primeiras aplicações do MEF ao cálculo de campos vetoriais EM, observou-se a ocorrência de modos espúrios. Ao longo das duas últimas décadas, vários autores têm procurado investigar as causas deste problema e buscado formas de garantir a confiabilidade da solução final.
As formulaçōes vetoriais mais utilizadas em problemas de guias de ondas são a equação de Helmholtz para as componentes longitudinais $E_{z}$ e $H_{z}$ (equação dos modos híbridos) e a equação vetorial de Helmholtz para as três componentes dos campos E ou H. Em 1970, Cendes e Silvester [12] relatam o surgimento de "soluçōes nãofísicas" na determinação dos modos de guias parcialmente cartegados com dielétricos, usando uma formulação variacional baseada na equação dos modos híbridos, e propõem a observação do comportamento dos campos como forma de distinguir entre modos físicos e espúrios. No contexto da equação vetorial de Helmholtz envolvendo as três componentes do campo, os modos espúrios começaram a ser estudados na segunda metade da década de 70. Em 1976, Konrad [13] apresenta os primeiros resultados para esta equação em que aparecem modos espúrios, reconhecendo-os por violarem a condição de divergência nula e nāo satisfazerem as condições de contorno.

A identificação a posteriori das soluções espúrias consiste em utilizar o funcional (13) sem modificaçāo e, a partir dos resultados, selecionar os autopares corretos através do pós-processamento dos autovetores ou por simples inspeção. O custo computacional elevado e a dificuldade na automatização desses procedimentos justificam a necessidade de se investigar métodos que possam garantir a priori a eliminação dos autopares espúrios. Tal objetivo pode ser alcançado por diversos procedimentos. que serão aqui agrupados em 3 categorias distintas. a saber :

1. Modificação do funcional : a idéla consiste em adicionar à formulação variacional correspondente à equação vetorial de Helmholtz um termo que incorpore, de alguma forma, a condição de solenoldahıdade. É possível, por exemplo, impor essa condição aproximadamente, através de multiplicaúres de Lagrange ou no sentido dos mínimos quadrados. Este último procedimento configura o método ds penalıdade $[5,6]$, que será tratado mais adiante;

2. Uso de outras formulaçōes variacionais : a necessidade de garantir soluções livres de moujos espúrios levou vários autores a investigar formulaçós variacionais alternativas. Hayata, Koshiba et al [9] expressaram. para problemas de guias de ondas, a componente $\mathrm{H}_{\mathrm{r}}\left(\mathrm{Ou} \mathrm{E}_{\mathrm{z}}\right)$ em termos de $\mathrm{H}_{x}$ e $\mathrm{H}_{y}$ (ou $E_{x}$ e $E_{y}$ ), através da própria equação da divergência discretizada. reduzindo o sistema global obtido pelo MEF a uma função apenas das componentes transversais e apresentaram resultados sem a presença de modos espúrios. Mais recentemente, Fernandez e Lu [14] também apresentaram resultados livres de modos espúrios, partindo das expressões gerais do campo em função das componentes transversais e obtendo uma formulação variacional em termos de $\mathrm{H}_{\mathrm{x}} \mathrm{e}$ $\mathrm{H}_{\mathrm{y}}$. Angkaew et alli [15], Chew e Nasir [16] e diversos outros autores desenvolveram formulações alternativas, mas todas, inclusive as aqui mencionadas, envolvem um acréscimo considerável de procedimentos computacionais em comparação com a formulação básica (13) ;

3. Uso de novos elementos : nesta categoria encontram-se os métodos que mantêm a formulação variacional básica e 
modificam a natureza das funções de base, criando novos tipos de elementos capazes de garantir um espaço de soluções com divergência nula. Aqui se incluem as abordagens de Crowley, Silvester e Hurwitz [17], Kobelansky e Webb [18] e Hano [19], entre outras. Os chamados elementos covariantes $[17,20]$ são elementos curvilíneos isoparamétricos que usam funções de base de ordens diferentes para as componentes transversal e longitudinal dos campos e são capazes de resolver problemas de geometria complexa. Kikuchi [21] utiliza os elementos de baixa ordem propostos por Nedelec [22] para garantir que todas as soluçōes numéricas sejam aproximações de modos físicos. Bossavit e Mayergoyz [23] discutem as vantagens de usar os chamados elementos de aresta em problemas de espalhamento. Recentemente, Aubourg e Guillon [24] usaram elementos mistos nas soluçōes de microstrips com perdas e afirmaram que eles coincidem com os elementos retangulares de Hano [19] e com a classe de elementos que Bossavit [25] gera através das formas de Whitney.

Dentre os vários procedimentos aqui mencionados, foram escolhidos para serem analisados e implementados 3 métodos bem distintos, que exemplificam cada uma das categorias acima descritas :

- o método da penalidade : o mais frequentemente utilizado dentre os métodos da categoria 1, tem uma formulação matemática abrangente e aplicação em diversas áreas da Engenharia. Neste trabalho, além do método de penalidade tradicional, será discutida e aplicada a problemas de guias de ondas dielétricos a técnica de integração reduzida seletiva [26], que melhora significativamente o desempenho do método, como será comprovado adiante;

- o método das componentes transversais de Hayata e Koshiba : dentro da categoria 2, seu grande atrativo é garantir a completa eliminação dos modos espúrios em todo o espectro com uma formulaçāo teórica simples;

- elementos de aresta : a mais recente das técnicas de eliminação de modos espúrios, propõe uma abordagem que embute as características físicas do campo eletromagnético (continuidade tangencial, descontinuidade normal) aos tipos de elementos e suas funções de interpolação.

A seguir, esses métodos para eliminação de modos espúrios serão apresentados e suas implementações no problema de guias de ondas dielétricos serão descritas na próxima seção.

\subsection{Método da Penalidade}

O método da penalidade, empregado inicialmente em aplicações do MEF a problemas de Mecânica e Análise Estrutural, consiste em acrescentar à formulação variacional básica do problema um termo que impõe a condiçāo de solenoidalidade no sentido dos mínimos quadrados, isto é :

$$
F_{p}(\mathbf{H})=F(H)+s P(H)
$$

onde $\mathrm{F}(\mathbf{H})$ é o funcional em (13)

$$
\mathrm{P}(\mathbf{H})=\int_{\Omega}\left(\operatorname{div} \mathbf{H}^{*} \cdot \operatorname{div} \mathbf{H}\right) d \Omega
$$

e $s$ é um número real positivo chamado constante de penalização - à medida que $s$ aumenta, cresce a restrição imposta aos modos espúrios [5]. A equação matricial global, obtida após a discretização de (21) pelo MEF, se escreve:

onde

$$
([S]+s[U]) \mathbf{h}-k_{0}^{2}[T] \mathbf{h}=0
$$

$$
[\mathrm{U}]=\sum_{\mathrm{e}} \int_{\Omega e} \mathrm{CC}^{\mathrm{T}} d \Omega \mathrm{e}
$$

é a matriz global correspondente ao termo de penalização e

$$
C=\left[\begin{array}{lll}
d N_{e} / d x & d N_{e} / d y & \beta N_{e}
\end{array}\right]^{T}
$$

onde $\mathrm{N}_{\mathrm{e}}$ é o vetor das funções de base. $\mathrm{Na}$ implementação do método da penalidade, que será descrita adiante, foram escolhidas funções de base Lagrangeanas de segunda ordem [11].

Os resultados publicados até o momento com o uso do método da penalidade em problemas de guias de ondas apresentam como principal limitação a incapacidade de eliminar os modos espúrios de uma região arbitrária do espectro. Se o valor de s for muito pequeno, vão aparecer espúrios na região de interesse, e se for grande, os autovalores físicos perdem precisão. $\mathrm{Na}$ verdade, para cada modo o valor ótimo de $\mathrm{s}$ é diferente, sendo impossível a determinação a priori deste valor para um dado intervalo de freqüências, o que compromete o desempenho global do método. Um procedimento simples que permite estipular o valor de $\mathbf{s}$ arbitrariamente grande, eliminando os espúrios do intervalo espectral de interesse e sem que a precisão dos modos físicos fique comprometida será apresentado a seguir.

\subsection{Penalidade com integração reduzida : o método RIP}

Idealmente, o coeficiente de penalização s deveria poder crescer livremente para que os modos nāo-físicos fossem afastados da regiāo espectral de interesse. Na prática, isto não é possível, e a discretização por elementos finitos de (21) não funciona a contento. O problema surge porque o funcional $\mathrm{P}(\mathbf{H})$ em (22) é um termo de penalização apropriado para o problema variacional contínuo, isto é, a determinação das funções que tornam o funcional $F(\mathbf{H})$ em (13) estacionário, com a restrição da divergência nula. Isso, entretanto, não garante que a aproximação por elementos finitos de $\mathrm{P}(\mathbf{H})$, para um tamanho de malha arbitrário, também seja um termo de penalização apropriado para $F_{\mathrm{p}}(\mathbf{H})$. Para estar qualificado a ser um funcional de penalização, $\mathrm{P}(\mathbf{H})$ precisaria ser positivo semi-definido [27] e, em geral, este aparece como positivo definido. O problema é, entāo, dito "trancado" (overconstrained ou locked). 
As integrais na aproximação por $\mathrm{EF}$ são normalmente calculadas por integração numérica e, quando os integrandos são polinômios, existem fórmulas que fornecem vaiores exatos para estas integrais. Foi constatado, no contexto de problemas de Análise Estrutural, que se a regra de integração utilizada para o termo de penalização for de ordem menor que a necessária para uma integração exata, o funcional discretizado $F_{p}(H)$ se torna positivo semi-definido, evitando o "trancamento" da solução [27]. O conceito de integração reduzida seletiva (onde apenas algum termo é integrado com ordem menor que a exata) aplicado ao método da penalidade - RIP (Reduced Integration Penalty) - foi inicialmente empregado por Doherty et alli [28] em problemas de Engenharia Civil.

Para ilustrar o procedimento do método RIP, denota-se por $I_{e}(f)$ a regra de quadratura usada para aproximar a integral da função $f$ em cada um dos $E$ elementos finitos que constituem a malha definida em $\Omega$, ou seja:

$$
I(f)=\sum_{e=1}^{E} I_{e} \text { (f) }
$$

onde

$$
I_{e}(f)=\sum_{l=1}^{L} p_{1} f\left(\xi_{1}^{e}\right), \quad 1<e<E
$$

e, portanto

$$
\text { Ie }(\mathrm{f})=\int_{\Omega_{e}} \mathrm{f} d \Omega
$$

onde $p_{1}>0$ são os pesos da integração e $\xi_{1}^{e}$ são os pontos da regra de quadratura de Gauss utilizada dentro de cada elemento [10]. Se $\mathrm{f}$ for polinomial, é possivel escolher uma ordem $L$ para a regra $I_{e}$ tal que a igualdade se verifique em (28), isto é, a integração seja exata. A aproximação do funcional (23) pelo método RIP consiste em encontrar os autopares $\left\{\left(\mathrm{k}_{0}{ }^{2}\right)_{\mathrm{h}}{ }^{\mathrm{s}}, \mathrm{v}_{\mathrm{h}}{ }^{\mathrm{s}}\right\} E \mathrm{R} \times \mathrm{V}_{\mathrm{h}}$ que tornam estacionários o funcional:

$$
\begin{aligned}
& \mathrm{F}_{\mathrm{z}}\left(\mathbf{v}_{\mathrm{h}}{ }^{\mathrm{s}}\right)=\int_{\Omega}\left[\left(\varepsilon^{-1} \operatorname{rot}_{\mathbf{v}_{\mathrm{h}}}{ }^{\mathrm{s}}, \operatorname{rot} \mathrm{w}_{\mathrm{b}}\right)-\left(\mathrm{k}_{0}{ }^{2}\right)_{\mathrm{h}}{ }^{\mathrm{s}}\left(\mathbf{v}_{\mathrm{h}}{ }^{\mathrm{s}}, \mathrm{w}_{\mathrm{h}}\right)\right] d \Omega+ \\
& +\operatorname{sI}\left(\operatorname{div} \mathbf{v}_{\mathrm{h}}{ }^{5}, \operatorname{div} \mathbf{w}_{\mathrm{h}}\right), \quad \forall \mathrm{w}_{\mathrm{h}} \mathrm{E} \mathbf{v}_{\mathrm{h}}
\end{aligned}
$$

sendo I uma regra de integração numérica uma ordem inferior à usada para calcular numericamente as outras integrais em (29). Com isso, a matriz de penalização [U] em (24) passa a ser positiva semi-definida, evitando o "trancamento" do sistema [27].

A grande vantagem de se usar RIP é que o valor do coeficiente de penalização $s$ pode ser pré-fixado arbitrariamente grande, garantindo um intervalo espectral livre de modos espúrios sem, entretanto, afetar substancialmente a precisão dos autovalores físicos. $\mathrm{O}$ método RIP introduz, assim, uma forma eficiente de garantir a eliminação dos modos espúrios do intervalo espectral de interesse, eliminando a necessidade de procurar um coeficiente de penalização ótimo.

\subsection{Método das Componentes Transversais de Hayata e Koshiba}

O método das componentes transversais, desenvolvido por Hayata e Koshiba [9], aqui designado por H-K, consiste em eliminar do funcional $F(\mathbf{H})$ em (13) a componente longitudinal $\mathrm{H}_{\mathrm{z}}$, utilizando para isto a condição div $\mathbf{H}=0$. Assumindo uma variação longitudinal da forma $\exp (-j \beta z)$, obtém-se daquela condição:

$$
\mathrm{H}_{\mathrm{z}}=(1 / \mathrm{j} \beta)\left(\partial \frac{\partial \mathbf{H}_{\mathrm{x}}}{\partial \mathrm{x}}+\frac{\partial \mathbf{H}_{\mathrm{y}}}{\partial \mathrm{y}}\right)
$$

Aplicando a técnica de Galerkin a esta relação e usando a expressão (14), após somar-se as contribuições das matrizes elementares é possível escrever o vetor de incógnitas nodais $\mathbf{h}$ como :

$$
\mathbf{h}=[D] \mathbf{h}_{\mathbf{l}}
$$

onde

$$
\begin{gathered}
\mathbf{h}_{t}=\left[\begin{array}{ll}
\mathbf{h}_{\mathrm{x}} & \mathbf{h}_{\mathbf{y}}
\end{array}\right]^{\mathrm{T}} \\
{[\mathrm{D}]=\left[\begin{array}{c}
{[\mathrm{I}]} \\
{\left[\mathrm{D}_{z}\right]^{-1}\left[D_{t}\right]}
\end{array}\right]}
\end{gathered}
$$

$$
\begin{gathered}
{[D z]=\sum_{e} \int_{\Omega e} N_{e} N_{e}{ }^{T} d x d y} \\
\left.\left[D_{1}\right]=\sum_{e} \iint_{\Omega e} N_{e} \frac{\partial N_{e}{ }^{\top}}{\partial x} N_{e} \frac{\partial N_{e}{ }^{T}}{\partial y}\right]
\end{gathered}
$$

e [I] é a matriz unitária. Substituindo-se (31) em (17) e pré-multiplicando por $[D]^{\mathrm{T}}$, obtém-se a equação matricial global que tem como incógnitas as componentes transversais $\mathbf{h}_{\mathbf{t}}$ :

$$
\begin{array}{ll}
\text { onde } & {\left[S_{k}\right] \mathbf{h}_{\mathbf{t}}-k_{0}{ }^{2}\left[T_{k}\right] \mathbf{h}_{\mathbf{t}}=0} \\
& {\left[S_{k}\right]=[D]^{T}[S][D]} \\
& {\left[T_{k}\right]=[D]^{T}[T][D]}
\end{array}
$$

Desta forma, a condição de solenoidalidade é imposta às soluções numéricas, os modos espúrios são eliminados de todo o espectro e o número de incógnitas nodais diminui de $1 / 3 \mathrm{em}$ relação ao sistema original. Perde-se, porém, a esparsidade nas matrizes $\left[S_{k}\right]$ e $\left[T_{k}\right]$, o que acarreta em um grande aumento na demanda computacional, como será visto na próxima seçāo.

\subsection{Elementos de Aresta}

A denominação "elementos vetoriais tangenciais" é usada para identificar uma classe de elementos finitos que se caracteriza por ter funções de base cujos parâmetros (graus de liberdade) estão associados às arestas e não aos vértices dos elementos, como nos elementos Lagrangeanos (ou nodais). Uma propriedade importante dessa classe é que a componente tangencial do campo é contínua através da fronteira entre elementos, mas a 


\section{G. Migliora \& M. B. F. Chaves, e H. J. C. Barbosa}

MÉTODOS DE ELIMINAÇĀO DE SOLUÇŌES ESPÚRIAS DE GUIAS DIELÉTRICOS POR ELEMENTOS FINITOS :

\section{COMPARAC̄ĀO ENTRE DESEMPENHOS COMPUTACIONAIS}

componente normal pode ser descontínua. Em termos dos campos eletromagnéticos, essa propriedade é extremamente conveniente, uma vez que permite impor aos vetores apenas a continuidade fisicamente requerida (tangencial), eliminando a exigência de continuidade normal

que também é imposta pelas funções de base Lagrangeanas.

Dentro da classe de elementos vetoriais tangenciais, o conjunto de mais baixa ordem é constituído pelos chamados elementos de aresta ( edge elements), que correspondem à familia de mais baixa ordem dos elementos introduzidos por Nedelec [22]. Neste trabalho foram utilizados os elementos triangulares de aresta, identificados por Bossavit [23, 25] com as formas diferenciais de Whitney de ordem 1.

Considerando um elemento triangular genérico de vértices 1,2 e 3 , a forma de Whitney de ordem 1 (ou elemento de aresta) associada à aresta $\{\mathrm{j}, \mathrm{k}\}$ é definida por:

$$
\mathbf{W}_{\mathrm{e}}^{\mathrm{i}}=\mathbf{W}_{\mathrm{e}}(\mathrm{j}, \mathrm{k})=\lambda_{\mathrm{j}} \operatorname{grad} \lambda_{\mathrm{k}}-\lambda_{\mathrm{k}} \operatorname{grad} \lambda_{\mathrm{j}}
$$

onde os índices $\mathrm{i}, \mathrm{j}$ e $\mathrm{k}$ assumem os valores 1,2 e 3 , ciclicamente com módulo 3 , e $\lambda_{j}=\lambda_{j}(x, y)$ é a coordenada baricêntrica relativa ao vértice $j$ de um ponto de coordenadas $(x, y)$ do elemento, isto é:

$$
\left[\begin{array}{l}
\lambda_{1} \\
\lambda_{2} \\
\lambda_{3}
\end{array}\right]=\left[\begin{array}{ccc}
x_{1} & x_{2} & x_{3} \\
y_{1} & y_{2} & y_{3} \\
1 & 1 & 1
\end{array}\right]^{-1}\left[\begin{array}{l}
x \\
y \\
1
\end{array}\right]
$$

sendo $x_{j}$ e $y_{j}$ as coordenadas do vértice $j$. De (39) e (40), vem:

$$
\mathbf{W}_{\mathrm{e}}^{\mathrm{i}}=\left(1 / 2 \mathrm{~A}_{\mathrm{e}}\right)\left[\left(\mathrm{y}_{\mathrm{i}}-\mathrm{y}\right) \mathbf{a}_{\mathrm{x}}+\left(\mathrm{x}-\mathrm{x}_{\mathrm{i}}\right) \mathbf{a}_{\mathrm{y}}\right]
$$

onde $\mathbf{a}_{\mathbf{x}}$ e $\mathbf{a}_{\mathbf{y}}$ são os vetores unitários nas direçōes $\mathrm{x}$ e $\mathrm{y}$ e $A_{c}$ é a área do elemento triangular. As duas propriedades fundamentais destes elementos são as seguintes:

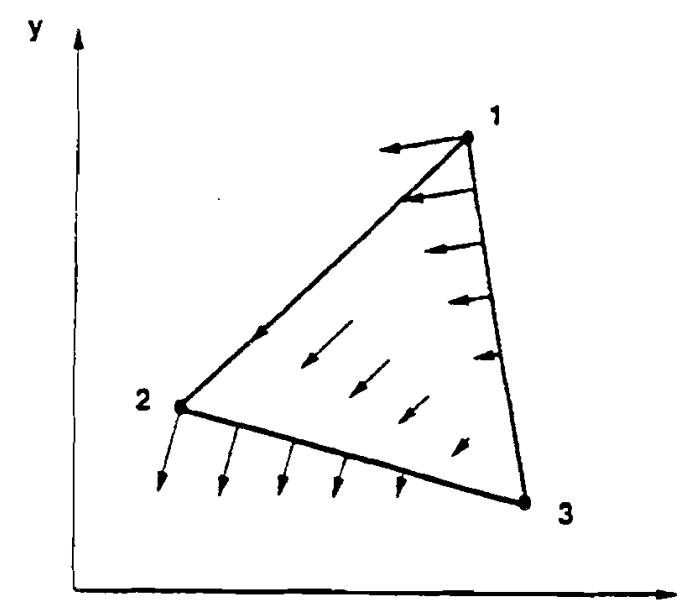

Figura 2 - Campo Vetorial $\mathrm{W}_{\mathrm{e}}(1,2)$ do elemento de Whitney.
1) a integral de linha de $\mathbf{W}_{\mathbf{e}}^{\mathbf{i}}$ tem valor 1 ao longo da aresta $\{j, k\}$ e zero nas outras arestas do elemento;

2) a componente tangencial de $\mathbf{W}_{\mathrm{e}}^{\mathrm{i}}$ é contínua através das 3 arestas do elemento, tendo valor nulo nas arestas ligadas ao vértice $i$.

A representação de um campo vetorial por uma combinação linear destes elementos tem como graus de liberdade as chamadas variáveis de aresta, que estão associadas às integrais de linha do campo ao longo de cada uma das arestas do triângulo. A Figura 2 mostra o comportamento do campo vetorial $\mathbf{W}_{\mathbf{e}}(1,2)$. No caso dos guias de ondas tratados aqui, subdividindo a seção reta em elementos triangulares, o campo magnético em cada elemento pode ser decomposto em componentes ortogonais $\mathbf{H}_{z}$ e $\mathbf{H}_{t}$, sendo esta última a soma de $\mathrm{H}_{x}$ e $\mathrm{H}_{\mathrm{y}}$. Como a componente $\mathbf{H}_{\mathbf{z}}$ é sempre tangencial às interfaces entre materiais diferentes, ela deve ser contínua e pode ser aproximada por funçōes de base Lagrangeanas

$$
\mathbf{H}_{\mathbf{z}}=\mathrm{j} \mathbf{N}^{\mathbf{T}} \mathbf{h}_{\mathbf{z}}
$$

onde $h_{\mathbf{z}}$ é o vetor de incógnitas nodais de $\mathbf{H}_{\mathbf{z}}$ e $\mathbf{N}$ é o vetor formado pelas coordenadas baricêntricas definidas em (40). Por outro lado, a componente $\mathbf{H}_{\mathbf{t}}$ deve apresentar apenas continuidade tangencial nas interfaces entre materiais e, para assegurar esta característica, foram usados elementos de aresta na sua aproximação, de forma que as componentes transversais são expressas por:

onde

$$
\begin{aligned}
& \mathrm{H}_{\mathrm{x}}=\mathbf{P} \cdot \mathbf{h}_{\mathbf{t}} \\
& \mathrm{H}_{\mathrm{y}}=\mathbf{Q} \cdot \mathbf{h}_{\mathbf{t}}
\end{aligned}
$$

$P=\left(1 / 2 A_{e}\right)\left[\begin{array}{lll}-a_{1}+c_{1} y & a_{2}-c_{2} y & a_{3}-c_{3} y\end{array}\right]$

$Q=\left(1 / 2 A_{e}\right)\left[b_{1}-c_{1} x-b_{2}+c_{2} x-b_{3}+c_{3} x\right]$

sendo $a_{k}=L_{k J} y_{m}, b_{k}=L_{k l} x_{m}$ e $c_{k}=L_{k l}$, onde $k, 1$ e m progridem com módulo 3 , isto é, ciclicamente em torno dos vértices 1,2 e $3, L_{k}$ é o comprimento da aresta correspondente, $x_{k}$ e $y_{k}$ são as coordenadas dos vértices do elemento e $h_{\mathbf{l}}$ é o vetor formado pelas variáveis de aresta em cada elemento.

Definidas as funçōes de base para as 3 componentes de $\mathbf{H}$, a expressão final do campo magnético aproximado em cada elemento é obtida de (42), (43) e (44):

$$
H_{e}=\left[N_{w}\right] h_{w}
$$

onde

$$
\left[N_{w}\right]=\left[\begin{array}{cc}
P^{T} & 0 \\
Q^{T} & 0 \\
0 & j N^{T}
\end{array}\right]
$$

e $h_{w}=\left[\begin{array}{ll}h_{t} & h_{z}\end{array}\right]$ é o vetor formado pelas incógnitas nodais de $\mathbf{H}_{\mathbf{z}}$ e pelas variáveis de aresta associadas a $\mathbf{H}_{\mathbf{t}}$. A Figura 3 ilustra um elemento triangular típico que combina interpolação Lagrangeana para a componente longitudinal e elementos de aresta para as componentes transversais. A substituição de (47) em (13) resulta, após os 
procedimentos usuais do $\mathrm{MEF}$, na equação matricial global:

$$
\left[\mathrm{S}_{\mathrm{w}}\right] \mathbf{h}_{\mathrm{w}}-\mathrm{k}_{0}^{2}\left[\mathrm{~T}_{\mathrm{w}}\right] \mathbf{h}_{\mathrm{w}}=0
$$

Expressões explícitas para as matrizes globais $\left[S_{w}\right]$ e $\left[T_{w}\right]$ podem ser encontradas em [7].

Apesar do grande número de trabalhos que vêm sendo publicados utilizando as diversas formas de elementos tangenciais, ainda não foi estabelecida uma teoria consistente para justificar seu desempenho. Os elementos de aresta usados aqui, por exemplo, têm funçōes de base que garantem a solenoidalidade do campo em cada elemento, o que é diversas vezes citado como justificativa para a eliminação dos modos espúrios. Estas funçōes, no entanto, não asseguram div $\mathbf{H}=0$ globalmente. Além disso, já foram descritos elementos de aresta que não têm divergência nula e ainda assim eliminam os modos espúrios [29]. Outra discussão freqüente é a que trata dos efeitos de usar funções de base de ordens diferentes para as diferentes componentes do campo, num mesmo elemento. Muitos autores consideram essa propriedade essencial para a eliminação de espúrios, mas no caso de elementos quadrangulares, sua aplicação não é suficiente para garantir apenas soluções físicas no MEF [29]. $y_{1}$

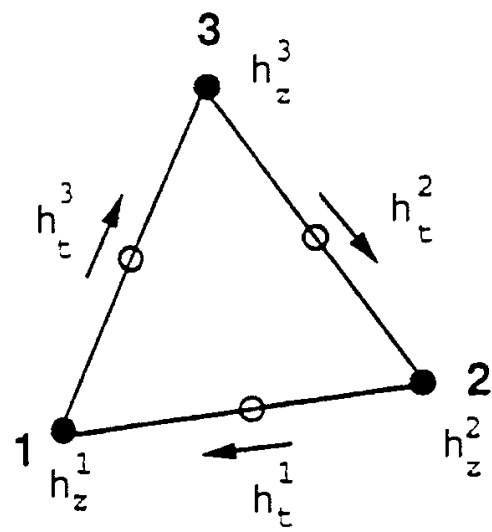

Figura 3 - Elemento Combinado Incógnitas Nodais Variáveis de Aresta.

\section{RESULTADOS NUMÉRICOS E CONCLUSÕES}

Tabela 1 - Autovalores $\mathrm{k}_{0}{ }^{2}$ de um guia de ondas oco de dimensões $7.112 \mathrm{~mm}$ e $3.556 \mathrm{~mm}$, para $\beta=0.5 \mathrm{rd} / \mathrm{m}$, obtidos cor formulação básica do MEF e com os métodos da penalidade (MP), RIP, H-K e ELA.

\begin{tabular}{|c|c|c|c|c|c|c|c|c|c|}
\hline Modo & $\begin{array}{l}\text { Sol. exata } \\
k^{2}\end{array}$ & $\begin{array}{c}\text { MEF básico } \\
\mathrm{k}_{0}{ }^{2}\end{array}$ & $\mathrm{k}_{0}^{2}{ }^{\mathrm{M}}$ & $\begin{array}{l}\text { P }(s=1.1) \\
\text { DIV }\end{array}$ & ROT & $\begin{array}{c}\mathrm{MP}(\mathrm{s}=100) \\
\mathrm{k}_{0}^{2}\end{array}$ & $\begin{array}{c}\operatorname{RIP}(s=100) \\
\mathrm{k}_{0}{ }^{2}\end{array}$ & $\begin{array}{r}\mathrm{H}-\mathrm{K} \\
\mathrm{k}_{0}{ }^{2}\end{array}$ & $\begin{array}{c}\text { ELA } \\
\mathrm{k}_{0}{ }^{2}\end{array}$ \\
\hline$S_{50}$ & & (35) & 0.2750 & 0.2500 & $0.71 \mathrm{E}-1$ & & & & \\
\hline $\mathrm{TE}_{10}$ & 0.4451 & 0.4452 & 0.4452 & $0.54 \mathrm{E}-4$ & 0.4452 & 0.4473 & 0.4472 & 0.4455 & 0.4476 \\
\hline$S_{10}$ & & (6) & 0.4897 & 0.4451 & $0.55 \mathrm{E}-4$ & & & & \\
\hline $\mathrm{TE}_{01}$ & 1.0305 & 1.0308 & 1.0308 & $0.88 E-4$ & 1.0307 & 1.0361 & 1.0349 & 1.0314 & 1.0403 \\
\hline $\mathrm{TE}_{20}$ & 1.0305 & 1.0355 & 1.0363 & 0.0014 & 1.0347 & 1.0896 & 1.0820 & 1.0368 & 1.0708 \\
\hline$S_{20}$ & & (2) & 1.1339 & 1.0307 & $0.97 \mathrm{E}-4$ & & & & \\
\hline $\mathrm{TE}_{11}$ & 1.2256 & 1.2262 & 1.2286 & $0.32 \mathrm{E}-3$ & 1.2283 & 1.2435 & 1.2365 & 1.2326 & 1.2359 \\
\hline$T M_{11}$ & 1.2256 & 1.2277 & 1.2288 & 0.0010 & 1.2276 & 1.2668 & 1.2558 & 1.2294 & 1.2626 \\
\hline$S_{11}$ & & (6) & 1.3514 & 1.2273 & 0.0013 & & & & \\
\hline $\mathrm{TE}_{21}$ & 1.8110 & 1.8228 & 1.8355 & 0.0017 & 1.8335 & 1.9365 & 1.8726 & 1.8609 & 1.8273 \\
\hline $\mathrm{TM}_{21}$ & 1.8110 & 1.8239 & 1.8376 & 0.0112 & 1.8253 & 2.0464 & 1.9638 & 1.8355 & 1.9610 \\
\hline$S_{21}$ & & (2) & 2.0185 & 1.8183 & 0.0180 & & & & \\
\hline $\mathrm{TE}_{30}$ & 2.0061 & 2.0593 & 2.0639 & 0.0139 & 2.0486 & 2.3540 & 2.2901 & 2.0632 & 2.2153 \\
\hline $\mathrm{S}_{30}$ & & (4) & 2.2676 & 2.0505 & 0.0121 & & & & \\
\hline $\mathrm{TM}_{31}$ & 2.7866 & 2.8657 & 2.9219 & 0.0504 & 2.8664 & 3.2590 & 2.9565 & 2.9077 & 2.8487 \\
\hline
\end{tabular}




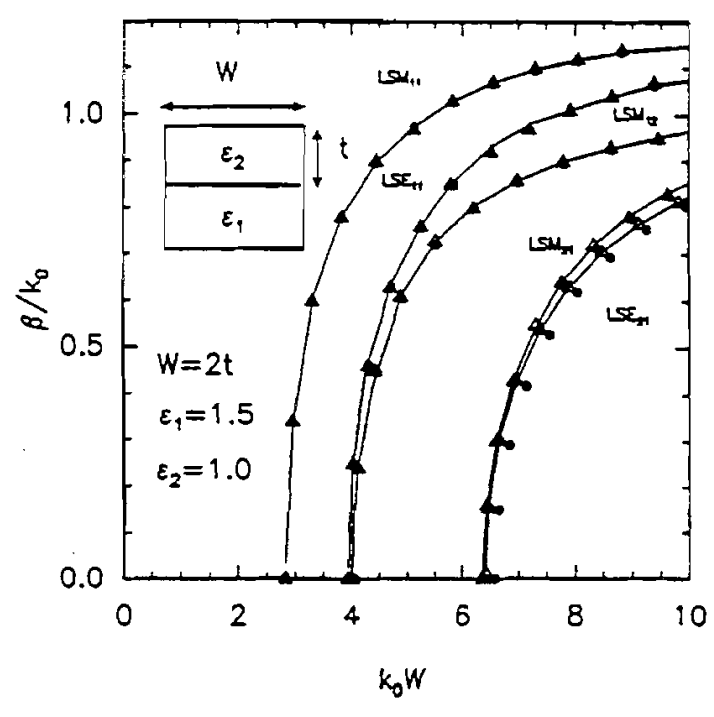

Figura 4- Diagrama de Dispersão de um Guia de Ondas Parcialmente Carregado, para $\beta=10 \mathrm{rd} / \mathrm{m}$ RIP $(s=100) \rightarrow$, HK e ELA •

Os resultados apresentados nesta seção foram obtidos de computaçōes efetuadas numa estação de trabalho IBM RISC-6000. Os problemas de autovalores expressos pelas equações matriciais globais obtidas na seção anterior foram resolvidos pelo método da iteração em subespaços, usando uma estrutura de dados de matrizes com armazenamento esparso. Elementos triangulares de segunda ordem foram empregados nas implementações dos métodos da penalidade (MP), penalidade com integração reduzida (RIP) e das componentes transversais de Hayata e Koshiba (H-K), com um esquema de integração numérica com 7 pontos de quadratura em cada triângulo, enquanto que no método RIP, a integração reduzida foi executada usando uma regra com 3 pontos por triângulo. $O$ método que emprega os elementos de aresta (ELA) foi implementado a partir das expressōes analíticas em [7], com as funções de base nodais de primeira ordem.

Na validação dos códigos computacionais desenvolvidos, foram considerados 2 aspectos relativos aos resultados obtidos : a verificação de que os autovalores são calculados corretamente e os modos espúrios, eliminados, e a taxa de convergência dos cálculos numéricos. No primeiro problema tratado, um guia de ondas retangular ôco, os resultados gerados com a formulação básica (17) do MEF comprovaram o aparecimento de vários modos espúrios, espalhados pela região espectral analisada, como se pode observar na Tabela $\mathbb{l}$, onde o número de espúrios (identificados por $S_{\mathrm{mn}}$ ) anteriores ao modo correto está entre parênteses. Estes modos apresentam elevados valores do parâmetro DIV (associado a div $\mathbf{H}$ ) e pequenos valores de ROT (associado a rot $\mathbf{H}$ ), ao contrário dos modos físicos. Com o acréscimo do termo de penalidade e um coeficiente $s=1.1$, o número de espúrios reduziu-se

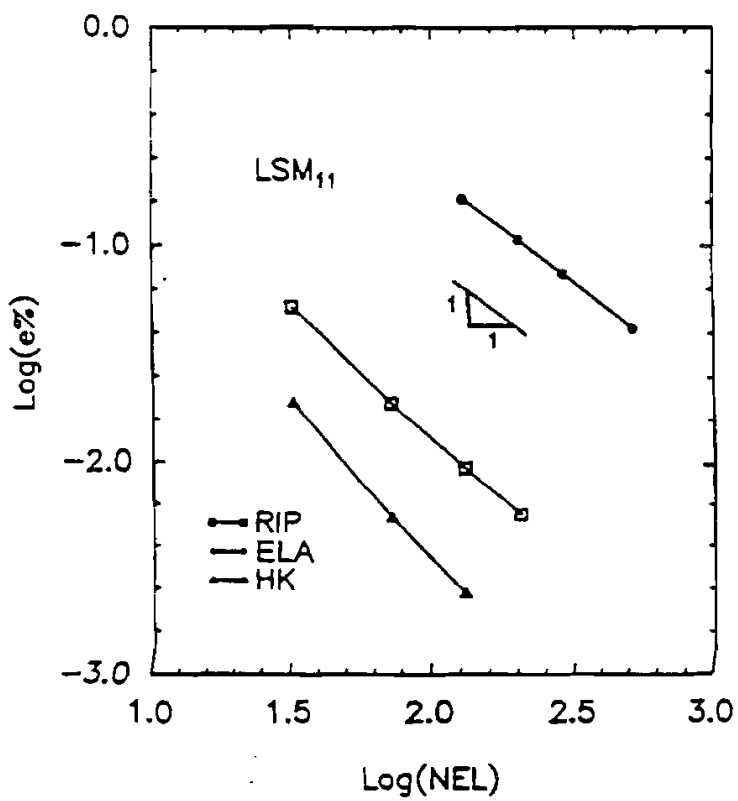

Figura 5- Convergência dos Autovalores do Guia Parcialmente Carregado $(\beta=10 \mathrm{rd} / \mathrm{m})$.

drasticamente, mas só com um valor de $s=100$ foi obtida a sua eliminação total até o modo $\mathrm{TM}_{31}$. Os métodos $\mathrm{HK}$ e ELA apresentaram resultados sem nenhum modo espúrio nessa faixa espectral .

O segundo caso analisado foi o de um guia quadrado carregado até a metade com um dielétrico de permissividade relativa 1.5. Novamente aqui, o uso da formulaçāo básica (17) gerou um grande número de espúrios na região espectral considerada. O método da penalidade com $s=1.1 \operatorname{logrou}$ remover a maioria destes. mas ainda restaram dois, identificados por $S_{00}$ e $S_{10}$. A Tabela 2, que compara os autovalores $k_{0}{ }^{2}$, para $\beta=10$ $\mathrm{rd} / \mathrm{m}$, obtidos pelos métodos MP, RIP, HK e ELA, com os fornecidos pela solução numérica das equações transcedentais em [30] com 6 casas decimais exatas (TRAN), mostra ainda que em todos os outros casos, a faixa do espectro considerada ficou livre de espúrios. Os resultados obtidos com o método RIP e $s=10000$ foram incluídos aqui para evidenciar a influência desprezível deste parâmetro sôbre a precisão dos autovalores quando se usa integração reduzida. Finalizando esta etapa da validação dos códigos, a Figura 4 mostra a excelente concordância nos diagramas de dispersão dos 5 primeiros modos do guia, obtidos pelos métodos RIP, HK e ELA.

A taxa de convergência dos resultados é um critério fundamental para se avaliar o desempenho de um método numérico e, no caso do MEF, esta é função da estratégia de incremento dos graus de liberdade. Um estudo sistemático da convergência das formulações descritas na seção anterior foi efetuado para os guias de ondas ôco e parcialmente carregado, usando a técnica de refinamento sucessivo da malha (convergência- $h$ ). As taxas de convergência obtidas com todos os métodos foram bastante próximas das previstas teoricamente $[7,31]$. A 
Figura 5 compara as convergências dos métodos RIP (com $s=10$ ), HK e ELA no cálculo dos autovalores $k_{o}$ (para $\beta=10 \mathrm{rd} / \mathrm{m}$ ) do modo $\mathrm{LSM}_{11}$ do guia parcialmente carregado, mostrando o erro relativo e\% $=100\left[\left(\mathrm{k}_{\mathrm{o}}-\mathrm{k}_{\mathrm{o}}{ }^{*}\right.\right.$ )$\left./ \mathrm{k}_{\mathrm{o}}{ }^{*}\right]$ no autovalor computado $\left(\mathrm{k}_{\mathrm{o}}{ }^{*}\right.$ é o autovalor com seis decimais exatas) em função do número (NEL) de elementos finitos $\mathrm{da}$ malha utilizada. $\mathrm{O}$ mesmo comportamento foi observado para outros valores de $\beta$ e também para o modo $\mathrm{LSE}_{1 \mathrm{I}}$, confirmando as taxas de convergência esperadas.

Uma vez validados os códigos computacionais e comprovada a convergência dos métodos investigados, foram analisadas algumas estruturas mais complexas. Primeiramente, foram calculados os modos de um guia de ondas retangular dielétrico, isotrópico, com permissividade relativa de 2.25 , envolvido pelo ar. A Figura 6 mostra as curvas de dispersão dos dois primeiros modos deste guia obtidas com os métodos RIP ( $s=100$ ), $\mathrm{H}-\mathrm{K}$ e ELA, podendo-se observar a excelente concordância entre estes resultados. Nenhum modo espúrio foi observado nestas computações.

A seguir, na Figura 7, mostra-se as características de dispersão dos dois primeiros modos de um guia de ondas retangular dielétrico, anisotrópico, obtidas com os três métodos de eliminação de espúrios considerados. Observa-se, novamente, uma excelente concordância entre os resultados, não tendo surgido nenhum modo espúrio nas computações. Para este exemplo e o anterior, foram utilizados guias de dimensões normalizadas 2 e 1 , e uma blindagem externa, perfeitamente condutora, de dimensões normalizadas 6 e 4, de modo a não interferir nos campos.

Vale ressaltar que as malhas de elementos finitos usadas nas análises dos guias metálicos ôco e parcialmente carregado foram estruturadas, enquanto que para os guias

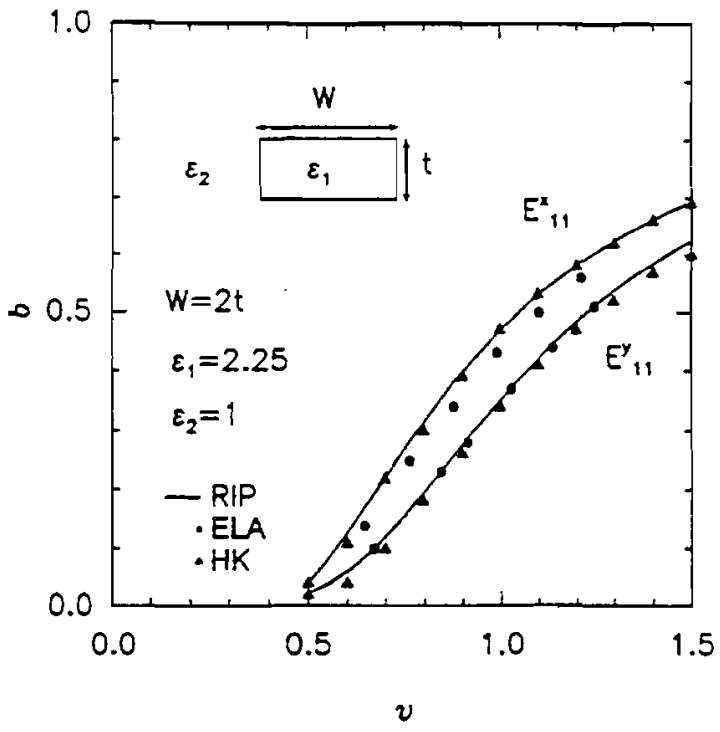

Figura 6- Características de Dispersão de um Guia Dielétrico Isotrópico (RIP com $s=1000$ ). $v=k_{0} t\left(\varepsilon_{1}-\varepsilon_{2}\right)^{1 / 2} / \pi$ e $b=\left[\left(\beta / k_{0}\right)^{2}-\varepsilon_{2}\right] /\left(\varepsilon_{1}-\varepsilon_{2}\right)$ dielétricos isotrópico e anisotrópico, foram nãoestruturadas. O gerador de malhas utilizado (ARANHA) emprega o algoritmo de Delaunay, em procedimentos adaptativos [7]. Foram testadas várias malhas, procurando-se aumentar o número de elementos nas regiões mais críticas de cálculo. Os resultados obtidos com os três métodos não demonstraram sensibilidade particular às variações de geometria das malhas. Este fator é muito mais marcante nas diferenças de precisão entre os modos, já que a malha escolhida pode ser mais apropriada para autovetores com determinadas características geométricas.

O cálculo dos autovalores do guia de ondas parcialmente carregado da Figura 4 foi usado como referência para comparar os desempenhos computacionais dos métodos de eliminação de espúrios. Como se sabe, o volume de cálculo numérico necessário para se resolver iterativamente um sistema linear é proporcional ao número (NZ) de elementos não-nulos da matriz global do sistema - mesmo no caso de métodos de solução direta, este é um fator crítico. A ura 8 mostra a variação de $\mathrm{NZ}$ em função do número (NGL) de graus de liberdade, ou incógnitas do sistema, para os métodos RIP (igual a MP), $\mathrm{H}-\mathrm{K}$ e ELA, evidenciando que o método RIP tem a vantagem, em relação ao $\mathrm{H}-\mathrm{K}$, de lidar com matrizes bem mais esparsas e apresentar a mesma taxa de variação que o método ELA, bastante inferior à do $\mathrm{H}-\mathrm{K}$.

A Tabela 3 mostra a ocupação de memória, em Kbytes, e o tempo de computação, em segundos, requeridos pelos três métodos para calcular os autovalores do guia parcialmente carregado, em termos do número de elementos na malha (NEL) e do número de graus de liberdade do sistema global (NGL). Tendo em vista que o método ELA apresenta interpolação de primeira ordem, diferentemente dos outros dois, que utilizam elementos de

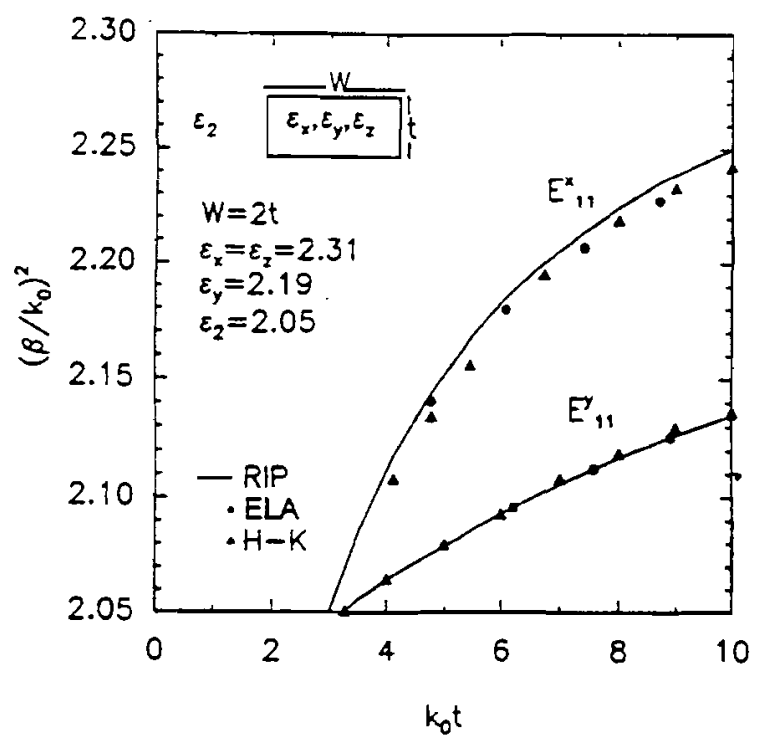

Figura 7- Característica de Dispersão de um Guia Dielétrico Anisotrópico (RIP com $s=1000$ ). 
segunda ordem, a comparação entre os desempenhos deve se basear no parâmetro NGL, mais diretamente relacionado aos requisitos de tempo e armazenamento do que o parâmetro NEL. Isto fica claro quando se observa na Figura 5 que o número de elementos necessários para se atingir uma mesma precisão nos autovalores é bastante diferente nos três métodos. Decidiu-se, então, plotar os erros relativos nos autovalores obtidos pelos três métodos em função da quantidade de memória (Kbytes) requerida nos cálculos. A Figura 9 mostra estes resultados para os dois primeiros modos do guia, podendo-se observar que o método RIP apresenta o melhor desempenho (menor ocupação de memória para uma mesma precisão) para o primeiro modo $\left(\mathrm{LSM}_{11}\right)$, e um desempenho intermediário para o segundo. Já o método ELA apresenta o pior desempenho para o primeiro modo e o melhor para o segundo. Isto se deve à configuração do campo magnético do modo $L_{S M} M_{1 !}\left(H_{y}=0\right)$, que não permite tirar proveito das características especiais dos elementos de aresta aqui implementados.

De posse dos resultados aqui apresentados, é possível concluir que o método $\mathrm{H}-\mathrm{K}$, apesar de fornecer a melhor precisão para uma mesma malha de EF, demanda o maior custo computacional para um dado grau de precisão. Sua implementação é trabalhosa, envolvendo a fatoração e a resoluçāo de vários sistemas matriciais, além da desvantagem de não ser aplicável a problemas estritamente tridimensionais como, por exemplo, ressoadores. Já o método ELA demanda os menores tempos de computação para uma dada precisão, mas sua implementação exige procedimentos não-usuais em $\mathrm{EF}$, que implicam em mudanças na estrutura de dados e na construção das matrizes. Este método também não é aplicável a problemas tridimensionais, apesar de haver outras implementações de elementos de arestas que o são.

Finalmente, o método RIP apresenta taxas de convergência similares às do $\mathrm{H}-\mathrm{K}$, com a vantagem de lidar com matrizes esparsas, exigindo muito menos tempo de computação e quantidade de memória. Sua implementação utiliza elementos Lagrangeanos tradicionais, não implicando em alterações na estrutura de dados e nos procedimentos dos pacotes básicos, o que permite o uso direto das bibliotecas de EF já existentes. O método é, ainda, aplicável a problemas tridimensionais, sem qualquer alteração na sua formulação. Assim, o método RIP se apresenta como uma solução de compromisso entre a qualidade das aproximações obtidas com o método $\mathrm{H}-\mathrm{K}$ e a economia computacional do método ELA, resgatando a utilização do método da penalidade com os atrativos que esta técnica proporciona.

\section{REFERÊNCIAS}

[1] Silvester, P., "Finite Elements in Electrical Engineering: The First 50 Years", Giornate di Studio su il Metodo degli Elementi Finiti nelle Applicazioni Dell'Elletromagnetismo, Pisa, Itália, 1992

[2] Saad, S.M., "Review of Numerical Methods for the Analysis of Arbitrarily-Shaped Microwave and Optical Dielectric Waveguides", IEEE Trans. MTT 38, pp. 894-899, 1985

[3] Silvester, P., "Finite Element Solution of Homogeneous 'Waveguide Problems", Alta Frequenza, 38, pp. 313-317, 1969

[4] Silvester, P., "High-Order Polynomial Triangular Finite Elements for Potential Problems", International Journal of Engineering Science, vol. 7, pp. 849-861, 1969

[5] Rahman, B.M.A. e J.B. Davies, "Penalty Function Improvement of Waveguide Solutions by Finite

Tabela 2 - Autovalores $\mathrm{k}_{0}{ }^{2}$ do guia parcialmente carregado da Fig. 4 (com $W=1 \mathrm{~m}$ ), para $\beta=10 \mathrm{rd} / \mathrm{m}$, obtidos com os métodos MP, RIP, H-K e ELA e através de solução numérica com 6 casas decimais exatas (TRAN) .

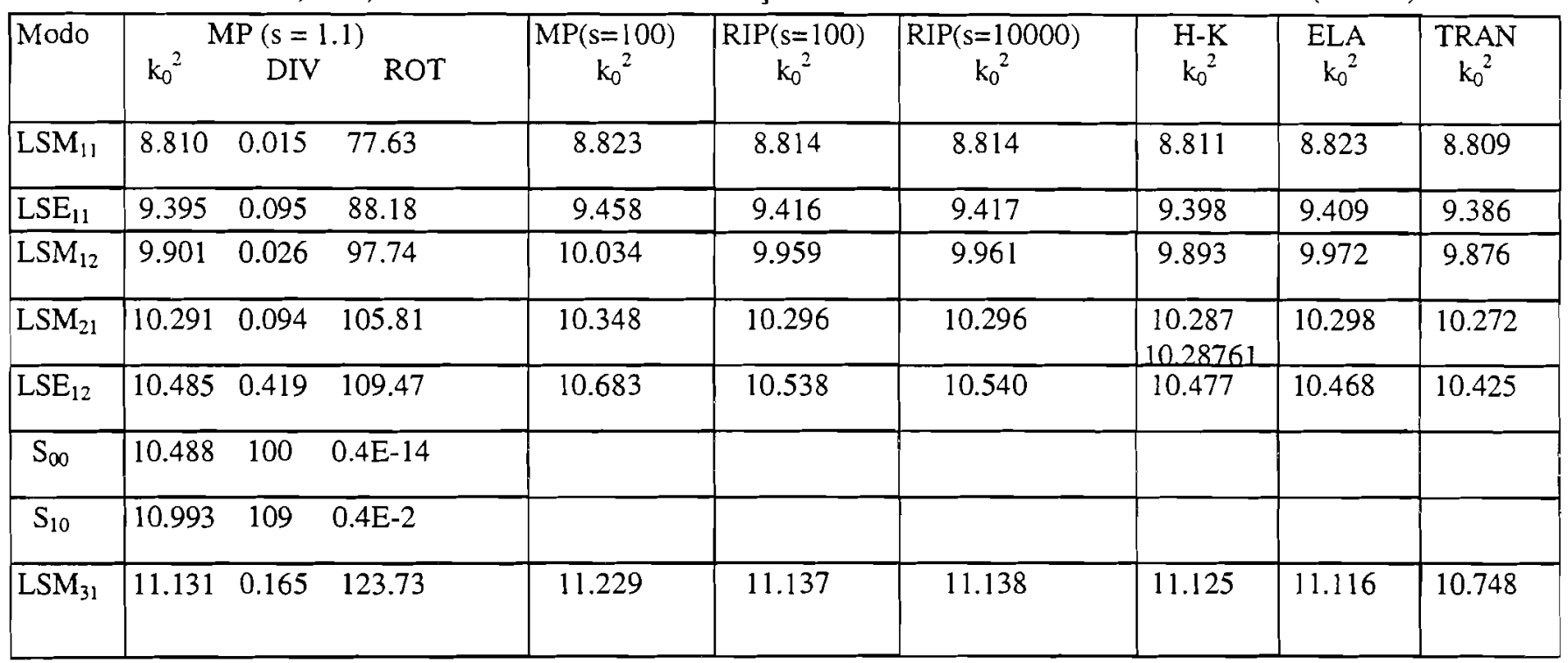




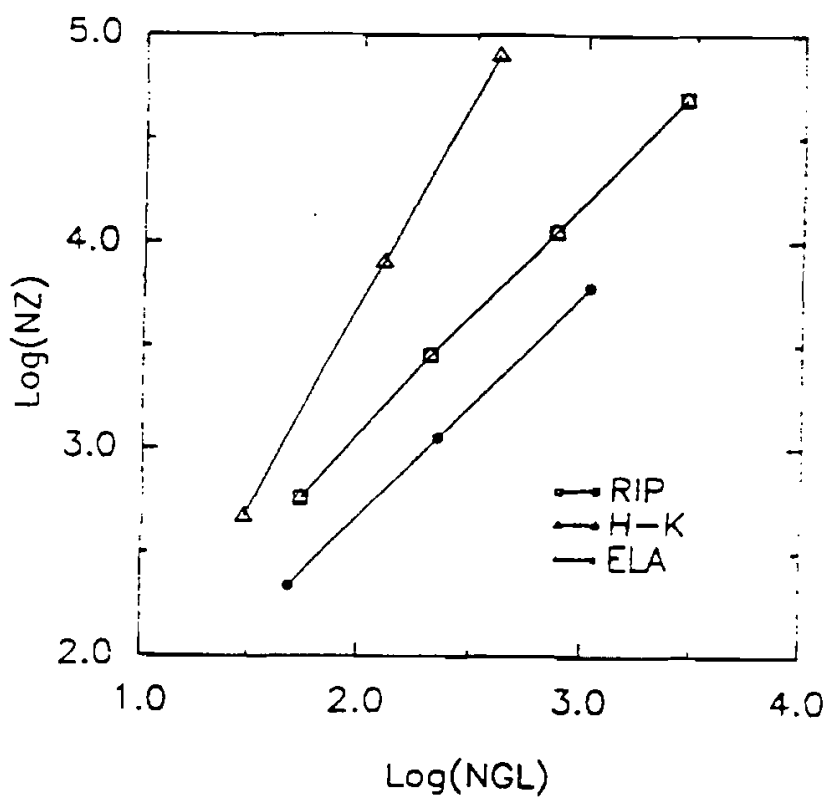

Figura 8- Número de Elementos não-nulos (NZ) na Matriz de EF em Função do Número de IGraus de Liberdade (NGL).

Elements", IEEE Trans. MTT - 32, pp. 922-928 , 1984

[6] Koshiba, M., K.Hayata e M.Suzuki, "Improved Finite Element Formulation in Terms of the Magnetic Field Vector for Dielectric Waveguides", IEEE Trans. MTT - 33, pp. 227-233, 1985

[7] Chaves, M.B.F., Eliminação de Modos Espúrios nas Soluções de Guias Dielétricos pelo Método de Elementos Finitos, Tese de Doutoramento, DEE, PUC-Rio, 1994

[8] Chaves, M.B.F., C.G.Migliora e H.J.Barbosa, "Elimination of Spurious FE Solutions of Dielectric Waveguides", COMPEL, vol. 13, suppl. A, pp. 323328,1994

[9] Hayata, K., M. Koshiba, M. Eguchi e M. Suzuki , "Vectorial Finite Element Method Without Any Spurious Solutions for Dielectric Waveguiding Problems Using Transverse Magnetic Field Component" , IEEE Trans. MTT - 34, 1986, pp.

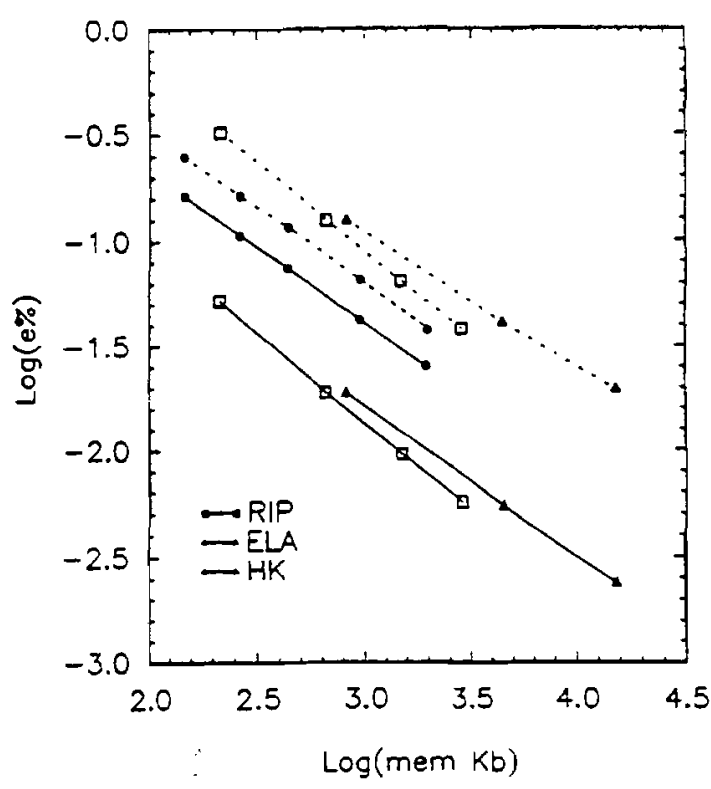

Figura 9- Erro Relativo nos Autovalores do Guia Parcialmente Carregado em Função da Quantidade de Memória Ocupada $\mathrm{LSM}_{11} \longrightarrow \mathrm{LSE}_{11} \ldots \ldots$.

$1120-1124$

[10] Zienkiewicz, O.C, The Finite Element Method Fundamentals and Applications, Academic Press, New York, 1973

[11] Silvester, P. e R. L. Ferrari, Finite Elements for Electrical Engineers, Cambridge Univ. Press, Cambridge , 1990

[12] Cendes, Z.J. e P. Silvester, "Numerical Solution of Dielectric Loaded Waveguide: I- Finite-element Analysis", IEEE Trans. MTT - 18, pp. 1124-1131, 1970

[13] Konrad A., "On the Reduction of the Number of Spurious Modes in the Vectorial Finite Element Solution of 3-Dimensional Cavities and Waveguides", IEEE Trans. MTT - 34, no. 2, pp. 224-226, 1986

[14] Fernandez , F.A. e Y. Lu, "A Variational Finite Element Formulation for Dielectric Waveguides in

Tabela 3 - Requisitos computacionais de tempo e memória para o cálculo dos autovalores do modo LSM $_{11}$ do guia parcialmente carregado da Fig. 4, pelos métodos RIP, H-K e ELA, em função do número de elementos da malha (NEL) e de graus de liberdade (NGL).

\begin{tabular}{|c|c|c|c|c|c|c|c|c|c|}
\hline NEL & \multicolumn{3}{|c|}{ NGL } & \multicolumn{3}{c|}{ Memória (em Kb) } & \multicolumn{3}{c|}{ Tempo (em seg.) } \\
\hline & RIP & H-K & ELA & RIP & H-K & ELA & RIP & H-K & ELA \\
\hline 32 & 211 & 162 & 81 & 218 & 825 & 29 & 2 & 34 & 1 \\
\hline 72 & 461 & 338 & 169 & 658 & 4483 & 71 & 9 & 170 & 2 \\
\hline 128 & 803 & 578 & 289 & 1490 & 15287 & 147 & 19 & 894 & 5 \\
\hline 200 & 1243 & 882 & 441 & 2840 & 28397 & 265 & 38 & $>1230$ & 8 \\
\hline 288 & 1779 & 1368 & 625 & 4831 & $>50000$ & 439 & 59 & & 23 \\
\hline
\end{tabular}


Terms of Transverse Magnetic Fields", IEEE Trans. Magnetic, vol. 27, pp. 3864-3867, 1991

[15] Angkaew, T., M. Matsuhara e N. Kumagai, "FiniteElement Analysis of Waveguide Modes: A Novel Approach That Eliminates Spurious Modes", IEEE Trans. MTT - 35, no. 2, pp. 117-123, 1987

[16] Chew, W.C. e M. A. Nassir, "A Variational Analysis of Anisotropic, Inhomogeneous Dieletric Waveguides", IEEE Trans. MTT - 37, pp. 661668", 1989

[17] Crowley, C. W., P. P. Silvester e H. Hurwitz, "Covariant Projection Elements for 3D Vector Field Problems", IEEE Trans. on Magnetics - 24, pp. 397-400, 1988

[18] Kobelansky, A. J. e J. P. Webb, "Eliminating Spurious Modes in Finite Element Waveguide Problems by Using Divergence-Free Fields", IEEE Trans. MTT - 22, no. 11, pp. 569-570, 1986

[19] Hano, M., "Finite Element Analysis of DielectricLoaded Waveguides", IEEE Trans. MTT - 32, no.10, pp.1275-1279, 1984

[20] Miniowitz, R. e J. P. Webb, "Covariant-projection Quadrilateral Elements for the Analysis of Waveguides with Sharp Edges", IEEE Trans. MTT 39, pp. 501-505, 1991

[21] Kikuchi, F., "Mixed and Penalty Formulations for Finite Element Analysis of an Eigenvalue Problem in Electromagnetism", Computer Meth. in Appl. Mech. and Eng., vol. 64, pp. 509-521, 1987

[22] Nedelec, J.C., " Mixed Finite Elements in $R^{3}$ ", Numer. Math., vol. 35, pp. 315-341, 1980

[23] Bossavit, A. e I. Mayergoyz, "Edge Element for Scattering Problems", IEEE Trans. on Magnetics 25, pp.2816-2821, 1989

[24] Aubourg, M. e P. Guillon, "A Mixed Finite Element Formulation for Microwave Devices Problems", Journal of Electromagnetic Waves and Applications, vol. 5 , no. 4 , pp. 371-386, 1991

[25] Bossavit, A., "Whitney Forms: a Class of Finite Elements for Three-Dimensional Computations in Electromagnetism", IEE Proceedings, vol. 135, pp. 493-500, 1988

[26] Malkus, D.S. e T. R. J. Hughes, "Mixed Finite Element Methods - Reduced and Selective Integration Techniques: A Unification of Concepts", Computer Methods in Applied Mechanics and Enginnering, vol.15, pp. 63-81, 1978

[27] Carey, G.F. e J. T. Oden, Finite Elements: A Second Course, Prentice - Hall, Englewood Cliffs, New Jersey, pp. 1120-1124, 1983
[28] Doherty, W.P., E. Wilson e R. Taylor, "Stress Analysis of Axisymmetric Solids Utilizing Higher Order Quadrilateral Finite Elements", SESM Report 69-3, Dept. of Civil Engineering, Univ. of California, Berkeley, 1969

[29] Dillon, B. M., P. Liu e J.P. Webb, "Spurious Modes in Quadrilateral and Triangular Edge Elements", COMPEL, vol. 13, suppl. A, pp. 311-316, 1994

[30] Collin, R. E., Field Theory of Guided Waves, Prentice - Hall, New Jersey, $2^{\text {nd }}$ Ed., pp. 411-416, 1991

[31] Chaves, M.B.F., C.G. Migliora e H. Barbosa, "Eliminating Spurious Solutions of Dielectric Waveguides : Computational Performance of the Reduced Integration Penalty Method", Proceedings of IEEE AP-S International Symposium, Newport Beach, California, pp. 68-71, 1995

Hélio J.C. Barbosa nasceu em Juiz de Fora, MG, em 1952. Formou-se em Eng. Civil pela Fac. Eng. da UFJF em 1974. Doutorou-se em Eng. Civil pela COPPE/UFRJ em 1986 e fez pós-doutorado na Division of Applied Mechanics da Stanford University. Trabalha no LNCC/CNPq e no Dept. de Ciência da Computação da UFJF desde 1980. Tem interesse em métodos numéricos e algoritmos genéticos para análise, otimização e identificação em engenharia.

Miriam B. F. Chaves nasceu em Penedo, Alagoas, em 3 de novembro de 1961. Concluiu o curso de Engenharia Elétrica na Universidade Santa Úrsula, em 1983 e obteve os graus de Mestre e Doutor em Ciências em Engenharia Elétrica na PUC-Rio em 1986 e 1994, respectivamente. Atualmente é Pesquisadora Assistente no Laboratório Nacional de Computação Científica do $\mathrm{CNPq}$, onde realiza pesquisas na área de métodos numéricos aplicados à propagação eletromagnética.

Carlos Gustavo Migliora nasceu no Rio de Janeiro em 18 de abril de 1952. Formou-se em Engenharia Elétrica (Telecomunicações) pela PUC-Rio em 1974 e obteve o grau de Mestre em Ciências em Engenharia Elétrica na mesma Universidade em 1976. Concluiu o doutoramento no Polytechnic Institute of New York (atualmente Polytechnic University), em 1980, com o grau de Ph.D. em Eletrofísica. Desde então leciona na PUC-Rio e trabalha em pesquisas no Centro de Estudos em Telecomunicaçōes da Universidade Católica (CETUC). Suas áreas de interesse são o Eletromagnetismo Aplicado. a propagação de ondas eletromagnéticas em meios naturais e os métodos numéricos aplicados à propagação eletromagnética. 